\title{
Large animal models for chronic wasting disease
}

\author{
C. K. Mathiason ${ }^{1}[$
}

Received: 2 November 2021 / Accepted: 19 January 2022 / Published online: 3 February 2022

(c) The Author(s), under exclusive licence to Springer-Verlag GmbH Germany, part of Springer Nature 2022

\begin{abstract}
Chronic wasting disease (CWD) is a fatal neurodegenerative prion disease of cervid species including deer, elk, moose and reindeer. The disease has shown both geographic and species expansion since its discovery in the late 1960's and is now recognized in captive and free-ranging cervid populations in North America, Asia and Europe. The facile transmission of CWD is unique among prion diseases and has resulted in growing concern for cervid populations and human public health. The development of native cervid host models with longitudinal monitoring has revealed new insights about CWD pathogenesis and transmission dynamics. More than 20 years of experimental studies conducted in these models, using biologically relevant routes of infection, have led to better understanding of many aspect of CWD infections. This review addresses some of these insights, including: (i) the temporal intra-host trafficking of CWD prions in tissues and bodily fluids, (ii) the presence of infectivity shed in bodily excretions that may help explain the facile transmission of CWD, (iii) mother-to-offspring CWD transmission, (iv) the influence of some Prnp polymorphisms on CWD susceptibility, and (vi) continued development of vaccine strategies to mitigate CWD.
\end{abstract}

Keywords Chronic wasting disease $\cdot$ CWD $\cdot$ Native host model

\section{Introduction}

The progressive geographical and host range expansion of CWD across North America, now into Asia and Europe, continues to elicit cervid and public health concerns. CWD poses a clear threat to cervid populations (Edmunds et al. 2016; DeVivo et al. 2017), and the zoonotic potential of CWD remains uncertain (Geist et al. 2017; Barria et al. 2011; Marsh et al. 2005; Comoy et al. 2015; Davenport et al. 2015; Ricci et al. 2017; Wang et al. 2021). The US Fish and Wildlife Service estimates that in 2016 alone, 9 million Americans ( 1 in 36) pursued big game such as deer and elk with $\sim 26$ billion spent on hunting (Service USFaW 2016). The human health risks from CWD are a growing concern in that it is estimated that between 7,000 and 15,000 CWD-infected cervids are consumed by humans annually (increasing 20\%/year) (Service USFaW 2016). To date, exposure to CWD has not been shown to result in human

C. K. Mathiason

candace.mathiason@colostate.edu

1 College of Veterinary Medicine and Biomedical Sciences, Department of Microbiology, Immunology and Pathology, Colorado State University, Fort Collins, CO, United States 80523 disease. However, given the long incubation periods of related human prion diseases (Prusiner 1982) and the lack of robust surveillance data for either human or animal populations, definitive conclusions are not possible.

\section{CWD discovery and spread over 40 years}

Chronic wasting disease (CWD) is a fatal neurodegenerative disease of cervid species (deer, elk, moose and reindeer) that has been identified in captive and free-ranging populations in North America, Korea and Scandinavia (Otero et al. 2021). CWD belongs to the larger family of protein misfolding disorders and is classified as a transmissible spongiform encephalopathy (TSE) or prion disease. The disease was first described in a population of mule deer that were not thriving in captivity (Williams and Young 1980). Originally, it was thought that the stress of captivity and nutritional deficits were the cause of their clinical presentation, which included lethargy and wasting. Post-mortem microscopic examination of brain tissue revealed the presence of lesions and vacuole formation similar to that reported in the brains of sheep with scrapie. These findings led to the diagnosis of a neurodegenerative disease, and the subsequent euonym naming of the condition as "chronic wasting disease." 
For over a decade after CWD's initial description, the focus was placed on identifying the disease through histological evaluation of post-mortem tissues of infected animals. These studies laid the important groundwork for identifying infected animals and disease progression. Field observations and studies revealed that CWD can be transmitted by direct animal-to-animal contact and by indirect contact with environments that previously housed CWD-infected cervids (Miller et al. 1998, 2004). The authors of these studies postulated that the infectious agent responsible for CWD may be present in bodily secretions, and that the agent was shed in sufficient quantity to initiate infection and disease progression among the herd. These studies also began to allude to the incredible resilience of the infectious prion in the environment, potentially contributing to its transmission in nature. The development and use of a novel CWD specific immunohistochemistry (IHC) assay (Spraker et al. 1997; O'Rourke et al. 1998) revealed CWD is efficiently transmitted within herds. Epidemiological evidence from captive animals suggests that horizontal transmission may occur at levels exceeding that observed in related prion diseases (Miller et al. 1998; Williams and Young 1992). These findings were surprisingly prescient, as CWD has recently been reported in captive herds with prevalence rates up to 80-90\% (Rivera et al. 2019), and in free-ranging cervid populations with prevalence as high as 30-50\% (Rivera et al. 2019). The consequence of this expansive disease burden is now resulting in cervid population decline (Edmunds et al. 2016; DeVivo et al. 2017).

Evidence supporting expanded geographic and cervid species distribution of CWD has been accumulating. Conventional prion detection methods (IHC, Western blot analysis, and ELISA) have been employed in lymphoid and brain tissue to probe for the presence of the partially proteinase resistant aberrant prion protein $\left(\mathrm{PrP}^{\mathrm{res}}\right)$, a biomarker of prion disease (CWD; $\mathrm{PrP}^{\mathrm{CWD}}$ ). Patterns of $\mathrm{PrP}^{\mathrm{CWD}}$ accumulation in cranial lymphoid tissues of preclinical cases provided the basis for an antemortem CWD test (Wild et al. 2002) and improved methods for large-scale CWD surveillance (Hibler et al. 2003). Prior to 2000, CWD was known to be present in 5 US States and 1 Canadian Province (Fig. 1). As of the writing of this review, CWD has been detected in 26 US States, 4 Canadian Provinces, South Korea, Norway, Finland and Sweden (Fig. 1) (Rivera et al. 2019; America USGSCmN 2021). Although the dramatic recording of CWD expansion may be in part due to greater surveillance and more reliable detection methods, it is apparent that the disease is spreading among cervid populations in North America and globally. Natural infections have been reported in a variety of cervid species, including mule deer (Odocoileus hemionus californicus), black-tailed deer (Odocoileus hemionus columbianus), white-tailed deer (Odocoileus virginianus), elk (Cervus canadensis), moose (Alces alces), red deer (Cervus elaphus elaphus) and reindeer (Rangifer tarandus tarandus). The well-described facile transmission of CWD led to further query as to how this disease is maintained within cervid populations.

\section{CWD infection progression from exposure, intra-host dissemination, to shedding}

Development of native host experimental model systems was critical to characterize the temporal distribution of CWD burden in tissues and secretions of infected cervids to determine their impact on CWD pathogenesis and transmission dynamics. Other non-familial TSEs, such as kuru, transmissible mink encephalopathy and bovine spongiform encephalopathy, have been shown to be transmitted via ingestion of infected tissues (Cervenakova et al. 1998; Marsh and Bessen 1993; Wells et al. 1998). Oral exposure is thus a plausible route of transmission of CWD prions for cervids in nature. In fact, oral inoculation has resulted in productive infections in moose (Kreeger et al. 2006), elk (Hamir et al. 2006; Plummer et al. 2017), white-tailed and mule deer (Plummer et al. 2017), red deer (Martin et al. 2009; Balachandran et al. 2010), sika deer (Cervus nippon) (Sohn et al. 2020) and reindeer (Mitchell et al. 2012). As further evidence to support the efficacy of oral infection and to track early CWD pathogenesis, an experimental study (Sigurdson et al. 1999) was conducted employing mule deer fawns captured outside the CWD endemic region (Miller et al. 2000; Miller and Williams 2003). The fawns were orally exposed to pooled brain homogenate harvested from naturally exposed CWD-infected mule deer. Conventional CWD detection methods (IHC) were used to demonstrate $\mathrm{PrP}^{\mathrm{CWD}}$ deposition within the retropharyngeal node, Peyer's patches and ileocaecal node as early as 1.5 months post-inoculation (mpi), and within tonsil as early as $2.75 \mathrm{mpi}$. These data were critical for providing: (i) proof of concept for CWD infection via oral exposure, and (ii) broad dissemination in lymphoid tissues adjacent to mucosal surfaces early in infection suggesting the possibility of prion shedding in bodily excretions (saliva, urine, feces).

A subsequent experimental study in mule deer using the same source inoculum, route of inoculation and CWD detection methodology as the above mule deer fawn study (Sigurdson et al. 1999) permitted clinical disease and $\mathrm{PrP}^{\mathrm{CWD}}$ dissemination monitoring throughout the disease course (inoculation to terminal clinical disease) (Fox et al. 2006). This study resulted in clinical manifestation consistent with CWD disease between 20 and 26 months post-oral exposure. Clinical presentation including dullness in eyes, diminished alertness, misdirected behaviors and piloerection occurred as early as 14-19 mpi. As clinical disease progressed, additional behavioral changes were noted. These changes included blank staring, uncharacteristic or subdued 
A

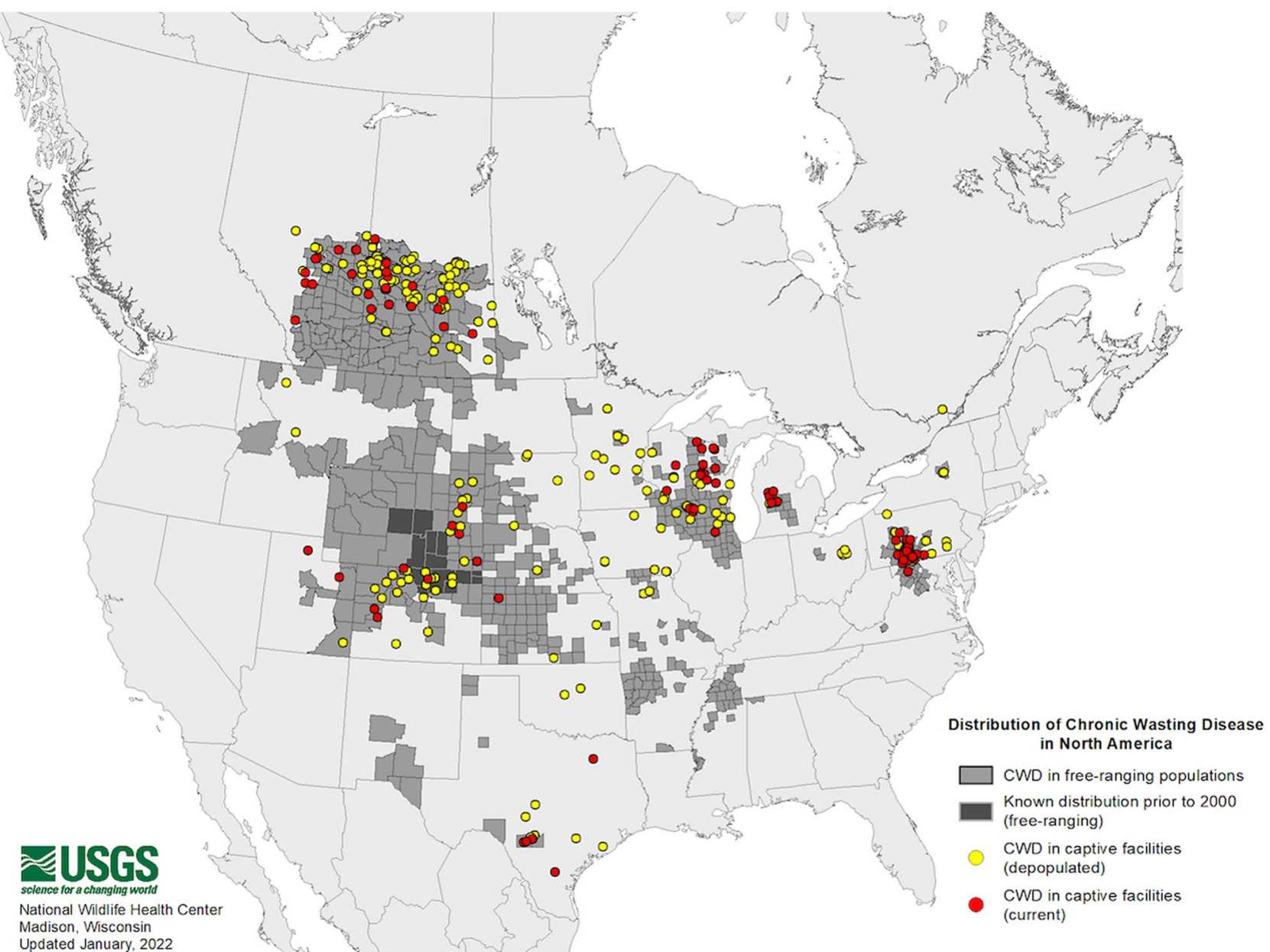

All locations are approximations based on best-available information

Fig. 1 Distribution of Chronic Wasting Disease in North America and Scandinavia. A. Distribution of Chronic Wasting Disease in North America. Credit and Source: Dr. Bryan Richards; National Wildlife Health Center, United States Geological Survey, Distribution of Chronic Wasting Disease in North America, https://www.usgs.gov/ media/images/distribution-chronic-wasting-disease-north-america-0,
January 2022. B. Distribution of Chronic Wasting Disease in Norway, Sweden and Finland. Credit and Source: Dr. Christer Rolandsen; The Norwegian information portal for deer management (www. hjortevilt.no) / Norwegian institute for nature research (www.nina. no). , October 26, 2021

tissue involvement revealed at $\sim 16$ mpi. These findings provided: (i) evidence that oral dosing resulted in similar timing and clinical presentation as described for natural CWD infections, (ii) refined detailing of early clinical disease manifestation and staging of clinical course, and (iii) evidence for and timing of $\operatorname{PrP}^{\mathrm{CWD}}$ distribution in a broad array of tissues.

Further exploration into how CWD is transmitted and sustained among native cervid populations was performed by a series of experimental studies conducted in cohorts of hand-raised and indoor adapted white-tailed deer (WTD). White-tailed deer were sourced from a region of the USA that to date is free of CWD (Georgia, USA). The controlled nature of housing permitted assessment of point source inocula, and temporal detection of CWD in serially collected at $~ 9$ mpi with more widespread CNS and neuroendocrine 


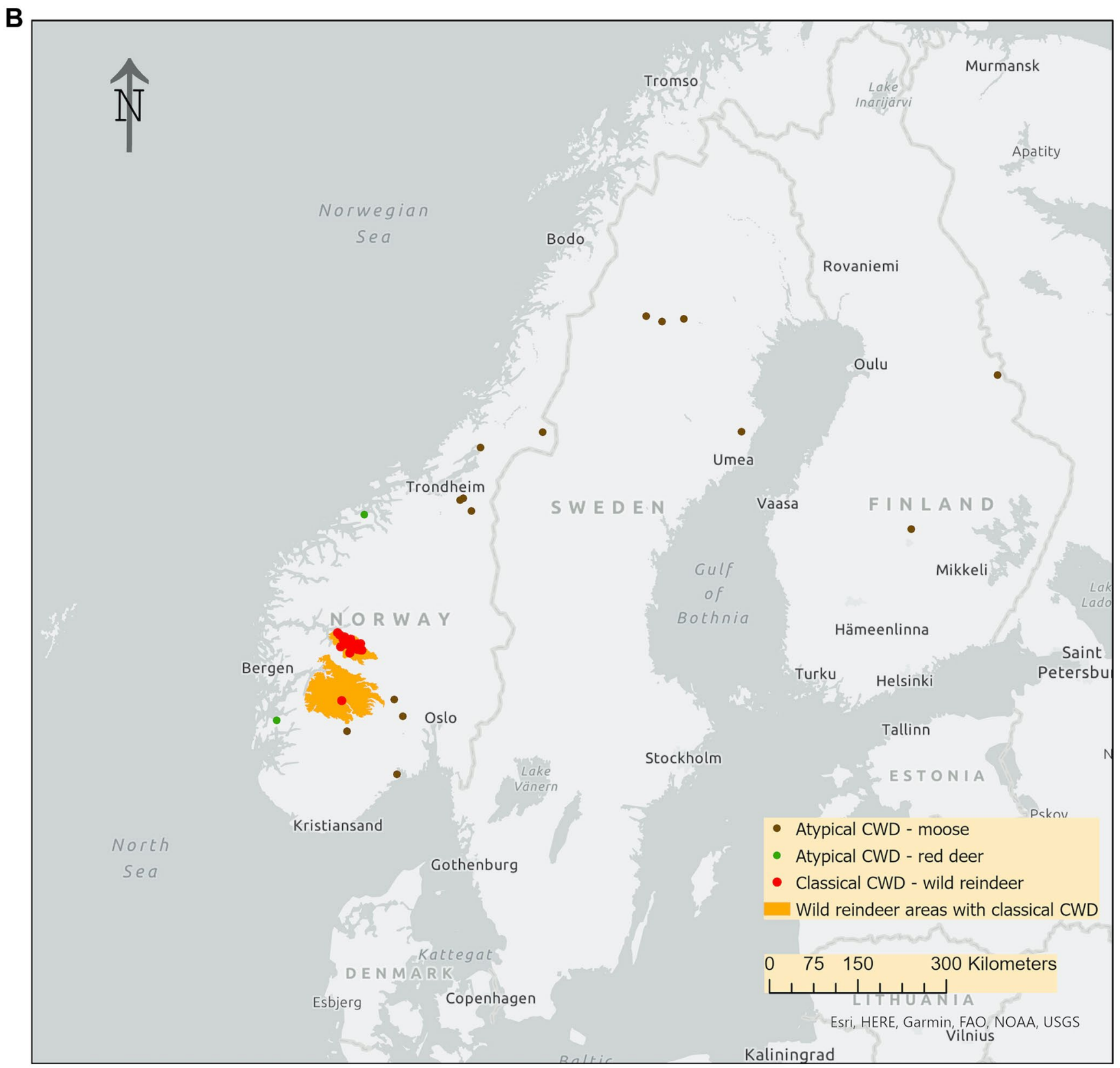

Fig. 1 (continued)

lymphoid biopsies and bodily excretions. This native host model provided a previously untenable level of longitudinal monitoring and evaluation of CWD transmission, prion dissemination and shedding.

Taking cues from natural infection observations (Miller et al. 2004), this WTD model was employed to assess the presence of infectivity in several bodily fluids and excretions (secreta)—saliva, urine, feces and blood. Although excretions were known to harbor infectious particles, it remained undefined which (saliva, urine, feces, blood) contained sufficient infectivity to initiate CWD infection. Cohorts of WTD were maintained in separate suites, permitting assessment of the infectious potential of saliva, urine, feces or blood (Mathiason et al. 2006). The inoculum was collected from clinical CWD positive naturally infected mule deer. Separate cohorts of WTD were orally dosed with saliva $(50 \mathrm{ml})$, or a combination of urine \& feces $(50 \mathrm{ml}$ or gr/each), or received a blood transfusion $(250 \mathrm{ml})$. As controls for infection, a cohort of WTD received orally dosed CWD positive brain (10 gr), and a cohort of WTD was orally dosed with brain, saliva, urine, feces and blood transfusion from confirmed CWD-negative WTD (same amounts and dosing as 
per above). Deer were monitored weekly for clinical signs of disease. Serial lymphoid (tonsillar and RAMALT) biopsies were taken at 3 month intervals to assess for the presence of PrP ${ }^{C W D}$ by IHC and Western blot analysis. Saliva, urine, feces and blood were collected at the same time interval, with each aliquoted and stored at $-80 \mathrm{C}$. The first lymphoid $\mathrm{PrP}^{\mathrm{CWD}}$ positivity was noted at $3 \mathrm{mpi}$ in WTD orally dosed with brain tissue; with all animals becoming lymphoid biopsy positive by $12 \mathrm{mpi}$. Animals inoculated with saliva or blood transfusion became lymphoid biopsy positive at $12 \mathrm{mpi}$, with demonstrable lymphoid $\operatorname{PrP}^{\mathrm{CWD}}$ deposition in all deer by 19 mpi. A subset of saliva and blood-inoculated WTD were further monitored for clinical disease onset. First signs consistent with CWD clinical presentation were identified between 15 and 19 mpi (staring, increased presence of whites of eye, behavioral changes) with noted progressive development (wide-leg stance, piloerection, polydipsia, polyuria) to terminal clinical presentation (leg lifting, head and neck arching) occurring between 24 and 33 mpi. None of the WTD orally inoculated with urine and feces resulted in $\mathrm{PrP}^{\mathrm{CWD}}$ detection in lymphoid tissue by conventional assay (IHC and Western blot) throughout the 19 month monitoring period. Interestingly, brain tissue harvested from this cohort was later evaluated by a sensitive mouse bioassay ( $\operatorname{Tg}($ CerPrP-E226)5037 (Angers et al. 2009)). Assessment revealed the presence of CWD infectivity (Haley et al. 2009). A follow-up bioassay study in the native host model was conducted to determine the presence of CWD infectivity in secreta shed by asymptomatic lymphoid biopsy positive WTD. The serial sample collections of saliva, urine, feces and blood harvested from deer in the initial study served as the point source inoculum for this second study (Mathiason et al. 2009). This set of WTD cohorts was inoculated as per the initial study. Interestingly, the saliva and blood harvested from the subclinical animals demonstrated similar time to lymphoid biopsy positivity as compared to the group that received saliva and blood from CWD-clinical animals. This suggests the duration of infectious shedding may not be confined to animals with obvious symptomatic infections. To complement these WTD studies to assess indirect transmission of CWD in the absence of direct animal-animal contact, a cohort of deer was exposed to bedding, water, and feed buckets (i.e., fomites) from suites containing CWD-lymphoid biopsy positive WTD (Mathiason et al. 2009). This cohort, exposed to CWD solely by indirect environmental exposure, demonstrated lymphoid $\mathrm{PrP}^{\mathrm{CWD}}$ deposition between 15 and 19 mpi. This study underlines the importance of the presence of maintained infectivity shed with secretions to the environment, and how they may play a role in the natural transmission of CWD. Taken together, these studies revealed that: (i) sufficient infectivity was present within brain, saliva and blood collected at clinical and preclinical stages of disease to initiate CWD infection that manifest in clinical TSE disease presentation similar to that observed in natural cervid infections (Miller et al. 2004; Mathiason et al. 2006), (ii) oral exposure to large volumes of urine and feces from CWD-infected cervids did not result in $\operatorname{PrP}^{\mathrm{CWD}}$ deposition or clinical manifestation at $19 \mathrm{mpi}$ (Mathiason et al. 2009), rather, yielded asymptomatic CWD infections (Haley et al. 2009), and (iii) that sufficient infectivity was present in fomites (hay, bedding, buckets, water) from CWD-infected WTD suites to initiate indirect (no direct physical contact) asymptomatic infections between 15 and 19 mpi (Mathiason et al. 2009).

With the infectious potential of secreta demonstrated, $\mathrm{PrP}^{\mathrm{CWD}}$ burden in oropharyngeal, urogenital and gastrointestinal tissue sources of excreta were evaluated. These tissues were evaluated by protein misfolding cyclic amplification (PMCA), an assay that allows amplification and detection of prion seeding activity in tissues harboring low concentrations of CWD (Saa et al. 2005). Low prion burdens were found in salivary gland, urinary bladder and distal intestinal tract tissues (Haley et al. 2011). These findings offer insights into the transmission dynamics of CWD as well as intrahost trafficking. Further insights examining early pathways of intra-host prion spread demonstrated $\operatorname{PrP}^{\mathrm{CWD}}$ and amyloid seeding activity in oropharyngeal lymphoid tissues as early as 1-month post-oral inoculation with $\mathrm{PrP}^{\mathrm{CWD}}$ levels present at 4 mpi approaching levels observed in terminal disease (Hoover et al. 2014). This study utilized another recent advancement in the detection of low prion burdens, real-time quaking induced conversion (RT-QuIC) (Atarashi et al. 2008). These results support early CWD replication in tissues that produce saliva that may contribute to shed prions in excreta. Together these WTD studies support the presence of amyloid seeding activity (RT-QuIC and PMCA) in serial collections of excreta (urine, saliva and feces) harvested across the longitudinal course of CWD infection, suggesting that CWD prions are intermittently shed in excreta during the full course of disease (Henderson et al. 2015a, 2017; Davenport et al. 2017; Tennant et al. 2020). These findings support earlier demonstration of the infectious agent in feces and saliva of CWD-infected asymptomatic free-ranging cervids (Tamguney et al. 2009, 2012).

The dissemination of CWD seeding activity to excretory tissues suggested robust intra-host CWD trafficking that remained understudied. Given the robust infectivity observed in WTD that received blood via transfusion, the role of hematogenous-borne prions was evaluated. Blood is a mixture of cellular and acellular components, and additional bioassay studies were conducted in WTD to evaluate which may harbor the infectious CWD agent. These studies indicated that CWD blood infectivity was associated with the cellular fraction of blood, with a significant role for B cells and platelets in trafficking CWD infectivity in vivo (Mathiason et al. 2010). These findings support earlier tissue-based studies associating putative follicular B 
cells with $\operatorname{PrP}^{\mathrm{CWD}}$ (Sigurdson et al. 1999). Taking advantage of the advances made in methods for in vitro detection of CWD seeding activity in blood, it was revealed that CWD prions cross the mucosal surface as early as 15 min postoral exposure (Elder et al. 2013; McNulty et al. 2020). This was followed by an eclipse phase and subsequent bi-motile presence of amyloid seeding activity between 2 and $3 \mathrm{mpi}$ and again near lymphoid biopsy positivity (McNulty et al. 2020; Elder et al. 2015). These findings suggest: (i) roles for specific blood cell subsets in prionemia, (ii) that hematogenous prions peripheralize early in infection extending to all parts of the body, which may result in (iii) new routes of neuroinvasion.

\section{CWD infectious dose and route of exposure}

This WTD experimental model has provided key insights into the spread of CWD in and between susceptible cervids. It has also identified likely sources for shed infectivity. Due to the tightly controlled housing and longitudinal monitoring developed with this model, it is also uniquely relevant to evaluate the minimum amount of peripherally shed infectious agent required to propagate CWD infection. Furthermore, this experimental model provided opportunity to assess whether acquiring peripherally shed prions, by single bolus or multiple low dose exposures, are important factors in CWD transmission. One study incorporated very low doses of saliva or brain (300 ng) in single- and multiple-dose regimens (Denkers et al. 2020). Oral exposures of brain or saliva — dosed as a single $300 \mathrm{ng}$ bolus, three $100 \mathrm{ng}$ doses, or ten $30 \mathrm{ng}$ doses, were delivered orally over 12 weeks. WTD cohorts receiving the single $300 \mathrm{ng}$ bolus and three $100 \mathrm{ng}$ dose became infected, while WTD cohorts dosed with ten $30 \mathrm{ng}$ doses remained negative. This study revealed: (i) that the minimum infectious CWD dose approximates 100-300 ng CWD positive brain or saliva equivalent, (ii) CWD infection appears to conform more with a threshold rather than cumulative dose dynamic, and (iii) that while time to first detection of infection was prolonged in low dose recipients, once infection was established no differences in disease pathogenesis were observed to higher dosed WTD.

The body of work conducted in natural and experimental settings clearly demonstrates that cervids are susceptible to oral CWD exposures, whether by direct (animal-to-animal) or indirect (environmental) contact with the infectious agent. Regardless of source and route, transmission of CWD prions via contact with the alimentary and/or respiratory mucous membranes seems assured. A study to determine the efficacy of respiratory mucosa exposure via aerosolization was undertaken in the WTD experimental system. It was found that this is an efficient mode of transmission ( $5 \mathrm{mg}$ brain tissue exposure), with infection kinetics equal to oral exposure (first biopsy positive at $3 \mathrm{mpi}$, with clinical presentation in all deer at $12 \mathrm{mpi}$ ) (Denkers et al. 2013). An additional study was conducted in WTD sourced from Missouri, USA, that were housed indoors (Nichols et al. 2013). The deer were inoculated by intranasal expulsion of lyophilized CWD positive brain $(200 \mathrm{mg}$ ) bound to montmorillonite clay $(250 \mathrm{mg})$. The study revealed that detectible $\mathrm{PrP}^{\mathrm{CWD}}$ deposition was present within lymphoid tissues as early as $\sim 3 \mathrm{mpi}$.

The housed WTD model of CWD has allowed for greater understanding of prion transmission, dissemination and shedding. Important facets of CWD pathology such as minimum infectious dose and alternative routes of infection continue to be explored. In addition to these important questions, this model has provided greater insight into other areas of CWD research such as: (i) CWD detection sensitivity (Haley et al. 2012; Henderson et al. 2013, 2020; Denkers et al. 2016), (ii) semi-quantitation of CWD loads (Henderson et al. 2015b), (iii) longitudinal assessments of CWD presence in saliva, urine, feces and alimentary tract, and third eyelid (Henderson et al. 2015a, 2017; Tennant et al. 2020; Cooper et al. 2019), (iv) the ability to use paraffin embedded tissues for the detection of CWD prions (Hoover et al. 2016) and, (v) direct comparison of CWD conventional, amplification and bioassay methods (McNulty et al. 2019).

\section{CWD vertical transmission}

Horizontal CWD transmission, whether by direct animalto-animal contact, or indirect contact with prion infectivity shed into the environment, has been well documented. Fewer studies have monitored the possibility of vertical transmission from mother-to-offspring. A small cervid model, the Reeves' muntjac deer, provided opportunity to assess timed pregnancies in dams with known CWD status. This cervid model is polyestrous, indoor maintainable, and has similar CWD susceptibility and pathogenesis as native mule deer and WTD (Nalls et al. 2013). Muntjac dams were orally or subcutaneously inoculated with CWD positive brain tissue, becoming lymphoid biopsy positive between 3 and 4 mpi. Naïve and CWD positive dams were bred by live coverage with naïve muntjac bucks. Fawns were live-birthed, or fetal tissues were harvested at specific time points across gestation (1st, 2nd and 3rd trimester). The studies demonstrated CWD transmission from mother-to-offspring leading to detection of $\mathrm{PrP}^{\mathrm{CWD}}$ deposition in lymphoid tissue of fawns born to CWD-infected dams as early as 40 days post-birth that resulted in progressive clinical CWD disease between 30 and 54 months post-birth (Nalls et al. 2013). PMCA seeding activity was present in fetuses as early as the first trimester of pregnancy (Nalls et al. 2013), with infectivity (demonstrated by $\mathrm{Tg}(\mathrm{CerPrP}-\mathrm{E} 226) 5037^{ \pm}$mouse bioassay) 
present in the pregnancy microenvironment (uterus, amniotic fluid and placentomes) of CWD-infected pre-symptomatic dams (Nalls et al. 2017). To determine the impact, mother-to-offspring transmission may play in native freeranging cervid populations in utero harvested fetal samples collected from elk and WTD in CWD-endemic areas were assessed for CWD by PMCA and RT-QuIC amplification assays. The presence of prion seeds was demonstrated in fetal tissues from free-ranging elk (Selariu et al. 2015) and WTD (Nalls et al. 2021; Bravo-Risi et al. 2021), as well as in male reproductive tissues and semen of WTD (Kramm et al. 2019) providing evidence that vertical and/or sexual transmission may play a role in the facile transmission of CWD.

\section{Genetic factors that influence CWD susceptibility}

The above outlines factors involved in the spread of CWD from animal to animal, both directly and indirectly. Yet, another important facet in CWD transmission is the susceptibility of the naïve host to acquire the disease. A host's Prnp genotype and the primary structure of the $\mathrm{PrP}^{\mathrm{C}}$ protein(s) encoded have been shown to contribute to varying degrees of susceptibility to species-specific prion disease, in some cases completely preventing infection. In free-ranging cervid populations, the prevalence of CWD is lower in populations expressing at least one copy of specific Prnp polymorphisms suggesting reduced or delayed susceptibility to infection (Robinson et al. 2012; Monello et al. 2017; Haley et al. 2019). Understanding the prevalence of these susceptible and resistant Prnp genotypes in free-ranging cervid populations would allow for greater understanding of transmission dynamics and susceptibility compared to cervid herds with known CWD prevalence (Arifin et al. 2020). Experimental studies conducted in the native host have provided improved understanding of the role these genotypes play in disease. To test the hypothesis that Prnp genotype affects CWD disease progression and susceptibility in WTD, an experimental oral infection was performed (Johnson et al. 2011). Deer fawns possessing Prnp polymorphisms H95 and S96 were orally inoculated to determine the genetic contribution on the rate of disease progression. The authors demonstrated that deer homozygous for the wild-type prion protein Q95G96 succumbed to CWD infection significantly earlier than deer heterozygous or homozygous for the H95/S96 prion allele. Similarly, oral infection of Rocky Mountain elk (Hamir et al. 2006) revealed that elk expressing Prnp codon 132MM or 132LM were susceptible to oral inoculation (23-40 mpi), while those expressing codon 132LL elk remained clinically normal for $4+$ years, developing clinical CWD disease between 59 and 64 mpi. This suggested that 132LL elk may have reduced susceptibility or prolonged incubation to
CWD infection. Further analysis of brain tissues from this study revealed differences in $\mathrm{PrP}^{\mathrm{CWD}}$ deposition location and abundance. Elk harboring the susceptible genotype showed greater prion burden and neuropil deposition. In contrast, elk with reduced susceptible genotype showed reduced burden and glial associated deposition. These findings demonstrate that elk with longer incubation times (132LL) carried lower $\mathrm{PrP}^{\mathrm{CWD}}$ burdens than those that progressed to terminal clinical presentation in a shorter timeframe (132MM or 132LM) (Moore et al. 2018).

Enhanced understanding of Prnp polymorphisms represents a powerful tool to combat TSEs, with a clear demonstration observed in the sheep prion disease, scrapie. Sheep flock improvement programs have successfully reduced the cases of classical scrapie by incorporating breeding strategies to select for Prnp resistant stock (Soto et al. 2021; Hagenaars et al. 2010). Experimental studies and field studies that continue to shed light on the presence of Prnp polymorphisms and associate CWD status will help guide efforts to manage CWD through selective breeding strategies (Haley et al. 2021).

\section{CWD vaccine development}

Understanding factors that drive transmission and intrahost dissemination are key to understanding CWD disease and how to potentially stem its spread. Currently, there is no vaccine or treatment for any prion disease, including CWD. The dilemma with immune system-mediated vaccine approaches is that prion diseases do not elicit a bona fide immune reaction since $\operatorname{PrP}^{\mathrm{C}}$ and $\mathrm{PrP}^{\mathrm{Sc}}$ have the same primary structure (Aguzzi 2003; Aucouturier and Carnaud 2002; Mabbott et al. 2018). Newly generated prions are recognized as self-proteins by the immune system, and as adaptive immunity is mainly based on linear epitopes, prion infections do not result in detectable humoral and cellular immune responses. As well, there is no evidence that $\mathrm{PrP}^{\mathrm{Sc}}$ represents a pathogen-associated molecular pattern for innate immunity, although microglia involvement has been described (Mabbott et al. 2018; Perry 2004). Nevertheless, it has been demonstrated that immunization approaches can be effective against prion infections (Enari et al. 2001; Peretz et al. 2001; Heppner et al. 2001; White et al. 2003).

Novel vaccines have been evaluated in three cervid species, two with CWD challenge. A recombinant prion protein vaccine (Abdelaziz et al. 2018) was assessed in reindeer to determine if homologous and aggregation-prone recombinant prion protein $(\mathrm{PrP})$ could overcome self-tolerance and induce autoantibody production against the cellular isoform of $\operatorname{PrP}^{\mathrm{C}}$. Reindeer were dosed three times with 500 ug of Mouse dimer (Mdi) or Deer dimer (Ddi) immunogen with 5uM CpG adjuvant via subcutaneous route. This pilot study 
resulted in appreciable humoral immune responses in reindeer vaccinated with Mdi or Ddi recombinant protein, and post-immune sera harvested from reindeer receiving the Ddi vaccination mitigated CWD propagation in cell culture. Further studies in a cervid challenge model will be necessary to determine if this vaccine is efficacious in the control of CWD. A second nonchallenge CWD vaccine platform was investigated in WTD. Here, the authors targeted epitopes whose exposure for antibody binding depends on diseaseassociated misfolding of $\operatorname{PrP}^{\mathrm{C}}$ into $\operatorname{PrP}^{\mathrm{Sc}}$ Taschuk et al. 2017). Their vaccine consisted of a replication-incompetent human adenovirus expressing a truncated rabies glycoprotein $\mathrm{G}$ recombinant fusion with the rigid loop epitope (hAd5:tgG-RL). Oral immunization lead to the induction of $\mathrm{PrP}^{\mathrm{Sc}}$ specific systemic and mucosal antibody responses. Again, further studies in a cervid CWD challenge model will be necessary. A third vaccine targeting a YYR diseasespecific epitope was evaluated in elk with natural exposure to CWD contaminated environment (Wood et al. 2018). Elk were dosed intramuscularly with $50 \mathrm{ug}$ of the recombinant YYR-Lkt fusion protein with Emulsigen D at day 0, day 42, and annually for three years. All vaccinated elk produced YYR-specific antibody titers. Yet, the mean survival time was significantly shorter for vaccinated (800 days) vs unvaccinated elk (1062 days) after they were exposed to CWD contaminated paddocks. A fourth vaccine incorporated mucosal exposure to an attenuated Salmonella expressing PrP in white-tailed deer (Goni et al. 2015). Once a mucosal response was established, the vaccinated deer were boosted orally and locally by application of polymerized recombinant PrP onto tonsils and rectal mucosa. The white-tailed deer were then challenged orally with CWD. A significant prolongation of the incubation period was noted in vaccinated (909 dpi) vs controls (602 dpi) with one (1/5) deer remaining CWD free both clinically and by lymphoid biopsy. Efforts to find an effective vaccination strategy for CWD may require combined vaccine approaches and the incorporation of low dose or natural challenge longitudinal cervid model studies to assess their efficacy.

The current promising approaches in CWD vaccinology employ insights into the structure of $\mathrm{PrP}^{\mathrm{Sc}}$ (Wille and Requena 2018), and the proof-of-concept that tolerance to $\operatorname{PrP}^{\mathrm{C}}$ can be overcome by active vaccination as described above (Abdelaziz et al. 2018). Analysis of $\operatorname{PrP}^{\mathrm{Sc}}$ allowed definition of structure-specific surface epitopes as vaccine targets (Fang et al. 2019, in preparation). Immunization with this rationally designed, structure-based antigen, generated immune responses that specifically recognized $\operatorname{PrP}^{\mathrm{Sc}}$ in brain homogenates from prion-infected animals and protects transgenic mice carrying different human PRNP mutations against their disease-causing effects (Fang et al. in preparation). The finding that tolerance to $\operatorname{PrP}^{\mathrm{C}}$ can be overcome by active vaccination in reindeer and in mouse models, resulting in robust humoral and cellular responses against $\operatorname{PrP}$ without inducing unwanted side effects (Abdelaziz et al. 2017, 2018; Gilch et al. 2003; Polymenidou et al. 2004; Kaiser-Schulz et al. 2007) provides a second promising avenue to an efficacious CWD vaccine. Induced anti-PrP antibodies were found to block prion infection in vitro and resulted in prolonged survival of CWD-infected transgenic mice by up to $70 \%$ (Abdelaziz et al. 2017, 2018). The accumulated preliminary findings from these two diverse vaccine approaches indicate feasibility, efficacy and safety for longitunidal assessment, singly and in combination, in the experimental native whitetailed deer system described earlier in this review.

\section{Summary}

Over two decades of experimental studies in the native, cervid host has provided new insights to CWD pathogenesis, transmission dynamics, the importance of Prnp polymorphisms in infection susceptibility and disease progression, and the development of CWD mitigation strategies. Furthermore, use of these model systems has resulted in improved CWD detection methodologies, allowing greater exploration of key mechanistic and societal impacts associated with prions; including the prion conversion event and strain development, the impacts CWD has on free-ranging cervid populations, herd management, hunting and cervid industries, and the potential of transspecies transmission events to species sympatric with cervids, including humans.

Acknowledgements The author expresses gratitude to Ms. Corey Simpson for creative compilation of journal articles, Drs. Joseph Westrich and Nathaniel Denkers for critical manuscript review, and Drs. Bryan Richards and Chister Rolandsen for permissions to share North American and Scandinavian CWD distribution maps.

\section{Declarations}

Conflict of interest The corresponding author states that there is no conflict of interest.

\section{References}

Abdelaziz DH, Thapa S, Abdulrahman B, Lu L, Jain S, Schatzl HM (2017) Immunization of cervidized transgenic mice with multimeric deer prion protein induces self-antibodies that antagonize chronic wasting disease infectivity in vitro. Sci Rep 7(1):10538

Abdelaziz DH, Thapa S, Brandon J, Maybee J, Vankuppeveld L, McCorkell R et al (2018) Recombinant prion protein vaccination of transgenic elk PrP mice and reindeer overcomes self-tolerance and protects mice against chronic wasting disease. J Biol Chem

Aguzzi A (2003) Prions and the immune system: a journey through gut, spleen, and nerves. Adv Immunol 81:123-171

America USGSCmN (2021) Distribution of chronic wasting disease in North America. https://www.usgs.gov/media/images/distributi on-chronic-wasting-disease-north-america-0. Accessed Oct 2021 
Angers RC, Seward TS, Napier D, Green M, Hoover E, Spraker T et al (2009) Chronic wasting disease prions in elk antler velvet. Emerg Infect Dis 15(5):696-703

Arifin MI, Staskevicius A, Shim SY, Huang YH, Fenton H, McLoughlin PD et al (2020) Large-scale prion protein genotyping in Canadian caribou populations and potential impact on chronic wasting disease susceptibility. Mol Ecol 29(20):3830-3840

Atarashi R, Wilham JM, Christensen L, Hughson AG, Moore RA, Johnson LM et al (2008) Simplified ultrasensitive prion detection by recombinant PrP conversion with shaking. Nat Methods 5(3):211-212

Aucouturier P, Carnaud C (2002) The immune system and prion diseases: a relationship of complicity and blindness. J Leukoc Biol 72(6): 1075-1083

Balachandran A, Harrington NP, Algire J, Soutyrine A, Spraker TR, Jeffrey M et al (2010) Experimental oral transmission of chronic wasting disease to red deer (Cervus elaphus elaphus): early detection and late stage distribution of protease-resistant prion protein. Can Vet J 51(2):169-178

Barria MA, Telling GC, Gambetti P, Mastrianni JA, Soto C (2011) Generation of a new form of human $\operatorname{PrP}(\mathrm{Sc})$ in vitro by interspecies transmission from cervid prions. J Biol Chem 286(9):7490-7495

Bravo-Risi F, Soto P, Eckland T, Dittmar R, Ramirez S, Catumbela CSG et al (2021) Detection of CWD prions in naturally infected white-tailed deer fetuses and gestational tissues by PMCA. Sci Rep 11(1):18385

Cervenakova L, Goldfarb LG, Garruto R, Lee HS, Gajdusek DC, Brown P (1998) Phenotype-genotype studies in kuru: implications for new variant Creutzfeldt-Jakob disease. Proc Natl Acad Sci U S A 95(22):13239-13241

Comoy EE, Mikol J, Luccantoni-Freire S, Correia E, Lescoutra-Etchegaray N, Durand V et al (2015) Transmission of scrapie prions to primate after an extended silent incubation period. Sci Rep 5:11573

Cooper SK, Hoover CE, Henderson DM, Haley NJ, Mathiason CK, Hoover EA (2019) Detection of CWD in cervids by RT-QuIC assay of third eyelids. PLoS One 14(8):e0221654

Davenport KA, Henderson DM, Bian J, Telling GC, Mathiason CK, Hoover EA (2015) Insights into Chronic Wasting Disease and Bovine Spongiform Encephalopathy Species Barriers by Use of Real-Time Conversion. J Virol 89(18):9524-9531

Davenport KA, Hoover CE, Bian J, Telling GC, Mathiason CK, Hoover EA (2017) PrPC expression and prion seeding activity in the alimentary tract and lymphoid tissue of deer. PLoS One 12(9):e0183927

Denkers ND, Hayes-Klug J, Anderson KR, Seelig DM, Haley NJ, Dahmes SJ et al (2013) Aerosol transmission of chronic wasting disease in white-tailed deer. J Virol 87(3):1890-1892

Denkers ND, Henderson DM, Mathiason CK, Hoover EA (2016) Enhanced prion detection in biological samples by magnetic particle extraction and real-time quaking-induced conversion. J Gen Virol 97(8):2023-2029

Denkers ND, Hoover CE, Davenport KA, Henderson DM, McNulty EE, Nalls AV et al (2020) Very low oral exposure to prions of brain or saliva origin can transmit chronic wasting disease. PLoS One 15(8):e0237410

DeVivo MT, Edmunds DR, Kauffman MJ, Schumaker BA, Binfet J, Kreeger TJ et al (2017) Endemic chronic wasting disease causes mule deer population decline in Wyoming. PLoS One 12(10):e0186512

Edmunds DR, Kauffman MJ, Schumaker BA, Lindzey FG, Cook WE, Kreeger TJ et al (2016) Chronic Wasting Disease Drives Population Decline of White-Tailed Deer. PLoS One 11(8):e0161127

Elder AM, Henderson DM, Nalls AV, Hoover EA, Kincaid AE, Bartz JC et al (2015) Immediate and ongoing detection of prions in the blood of hamsters and deer following oral, nasal, or blood inoculations. J Virol 89(14):7421-7424

Elder AM, Henderson DM, Nalls AV, Wilham JM, Caughey BW, Hoover EA et al (2013) In vitro detection of prionemia in TSEinfected cervids and hamsters. PLoS One 8(11):e80203
Enari M, Flechsig E, Weissmann C (2001) Scrapie prion protein accumulation by scrapie-infected neuroblastoma cells abrogated by exposure to a prion protein antibody. Proc Natl Acad Sci U S A 98(16):9295-9299

Fang A, Flores-Fernández JM, Rathod V, Tang X, Wille H (in preparation) A rationally designed, structure-based vaccine protects a transgenic mouse model of Gerstmann-Strausser-Scheinker disease for neurodegeneration. In

Fang AF, Flores-Fernández JM, Rathod V, Tang X, Wille H (2019) Innocuous, structured scaffolds for structure-based amyloid disease vaccines and antigens. In. Edited by Application USP

Fox KA, Jewell JE, Williams ES, Miller MW (2006) Patterns of PrPCWD accumulation during the course of chronic wasting disease infection in orally inoculated mule deer (Odocoileus hemionus). J Gen Virol 87(Pt 11):3451-3461

Geist V, Clausen D, Crichton V, Rowledge D (2017) The challenge of CWD: Isidious and dire. Living Legacy White Paper Series; Version 1.0(Alliance for Public Wildlife): 1-40

Gilch S, Wopfner F, Renner-Muller I, Kremmer E, Bauer C, Wolf E et al (2003) Polyclonal anti-PrP auto-antibodies induced with dimeric PrP interfere efficiently with PrPSc propagation in prioninfected cells. J Biol Chem 278(20):18524-18531

Goni F, Mathiason CK, Yim L, Wong K, Hayes-Klug J, Nalls A et al (2015) Mucosal immunization with an attenuated Salmonella vaccine partially protects white-tailed deer from chronic wasting disease. Vaccine 33(5):726-733

Hagenaars TJ, Melchior MB, Bossers A, Davidse A, Engel B, van Zijderveld FG (2010) Scrapie prevalence in sheep of susceptible genotype is declining in a population subject to breeding for resistance. BMC Vet Res 6:25

Haley N, Donner R, Merrett K, Miller M, Senior K (2021) Selective Breeding for Disease-Resistant PRNP Variants to Manage Chronic Wasting Disease in Farmed Whitetail Deer. Genes (Basel) 12(9)

Haley NJ, Mathiason CK, Carver S, Telling GC, Zabel MD, Hoover EA (2012) Sensitivity of protein misfolding cyclic amplification versus immunohistochemistry in ante-mortem detection of chronic wasting disease. J Gen Virol 93:1141-1150

Haley NJ, Mathiason CK, Carver S, Zabel M, Telling GC, Hoover EA (2011) Detection of chronic wasting disease prions in salivary, urinary, and intestinal tissues of deer: potential mechanisms of prion shedding and transmission. J Virol 85(13):6309-6318

Haley NJ, Mathiason CK, Zabel MD, Telling GC, Hoover EA (2009) Detection of sub-clinical CWD infection in conventional testnegative deer long after oral exposure to urine and feces from CWD+ deer. PLoS One 4(11):e7990

Haley NJ, Merrett K, Buros Stein A, Simpson D, Carlson A, Mitchell $\mathrm{G}$ et al (2019) Estimating relative CWD susceptibility and disease progression in farmed white-tailed deer with rare PRNP alleles. PLoS One 14(12):e0224342

Hamir AN, Gidlewski T, Spraker TR, Miller JM, Creekmore L, Crocheck $\mathrm{M}$ et al (2006) Preliminary observations of genetic susceptibility of elk (Cervus elaphus nelsoni) to chronic wasting disease by experimental oral inoculation. J Vet Diagn Invest 18(1):110-114

Henderson DM, Davenport KA, Haley NJ, Denkers ND, Mathiason CK, Hoover EA (2015b) Quantitative assessment of prion infectivity in tissues and body fluids by real-time quaking-induced conversion. J Gen Virol 96(Pt 1):210-219

Henderson DM, Denkers ND, Hoover CE, Garbino N, Mathiason CK, Hoover EA (2015a) Longitudinal detection of prion shedding in saliva and urine by chronic wasting disease-infected deer by realtime quaking-induced conversion. J Virol 89(18):9338-9347

Henderson DM, Denkers ND, Hoover CE, McNulty EE, Cooper SK, Bracchi LA et al (2020) Progression of chronic wasting disease in white-tailed deer analyzed by serial biopsy RT-QuIC and immunohistochemistry. PLoS One 15(2):e0228327 
Henderson DM, Manca M, Haley NJ, Denkers ND, Nalls AV, Mathiason CK et al (2013) Rapid antemortem detection of CWD prions in deer saliva. PLoS One 8(9):e74377

Henderson DM, Tennant JM, Haley NJ, Denkers ND, Mathiason CK, Hoover EA (2017) Detection of chronic wasting disease prion seeding activity in deer and elk feces by real-time quakinginduced conversion. J Gen Virol 98(7):1953-1962

Heppner FL, Musahl C, Arrighi I, Klein MA, Rulicke T, Oesch B et al (2001) Prevention of scrapie pathogenesis by transgenic expression of anti-prion protein antibodies. Science 294(5540):178-182

Hibler CP, Wilson KL, Spraker TR, Miller MW, Zink RR, DeBuse LL et al (2003) Field validation and assessment of an enzyme-linked immunosorbent assay for detecting chronic wasting disease in mule deer (Odocoileus hemionus), white-tailed deer (Odocoileus virginianus), and Rocky Mountain Elk (Cervus Elaphus Nelsoni). J Vet Diagn Invest 15(4):311-319

Hoover CE, Davenport KA, Henderson DM, Pulscher LA, Mathiason CK, Zabel MD et al (2016) Detection and quantification of CWD prions in fixed paraffin embedded tissues by real-time quakinginduced conversion. Sci Rep 6:25098

Hoover CE, Denkers ND, Henderson DM, Soto C, Mathiason CK, Zabel MD et al (2014) Early prion trafficking in deer exposed to chronic wasting diseases. Prion 8:85

Johnson CJ, Herbst A, Duque-Velasquez C, Vanderloo JP, Bochsler P, Chappell R et al (2011) Prion protein polymorphisms affect chronic wasting disease progression. PLoS One 6(3):e17450

Kaiser-Schulz G, Heit A, Quintanilla-Martinez L, Hammerschmidt F, Hess S, Jennen L et al (2007) Polylactide-coglycolide microspheres co-encapsulating recombinant tandem prion protein with CpG-oligonucleotide break self-tolerance to prion protein in wildtype mice and induce CD4 and CD8 T cell responses. J Immunol 179(5):2797-2807

Kramm C, Gomez-Gutierrez R, Soto C, Telling G, Nichols T, Morales $\mathrm{R}$ (2019) In Vitro detection of Chronic Wasting Disease (CWD) prions in semen and reproductive tissues of white tailed deer bucks (Odocoileus virginianus). PLoS One 14(12):e0226560

Kreeger TJ, Montgomery DL, Jewell JE, Schultz W, Williams ES (2006) Oral transmission of chronic wasting disease in captive Shira's moose. J Wildl Dis 42(3):640-645

Mabbott NA, Alibhai JD, Manson J (2018) The role of the immune system in prion infection. Handb Clin Neurol 153:85-107

Marsh RF, Bessen RA (1993) Epidemiologic and experimental studies on transmissible mink encephalopathy. Dev Biol Stand 80:111-118

Marsh RF, Kincaid AE, Bessen RA, Bartz JC (2005) Interspecies transmission of chronic wasting disease prions to squirrel monkeys (Saimiri sciureus). J Virol 79(21):13794-13796

Martin S, Jeffrey M, Gonzalez L, Siso S, Reid HW, Steele P et al (2009) Immunohistochemical and biochemical characteristics of BSE and CWD in experimentally infected European red deer (Cervus elaphus elaphus). BMC Vet Res 5:26

Mathiason CK, Hayes-Klug J, Hays SA, Powers J, Osborn DA, Dahmes $\mathrm{SJ}$ et al (2010) B cells and platelets harbor prion infectivity in the blood of deer infected with chronic wasting disease. J Virol 84(10):5097-5107

Mathiason CK, Hays SA, Powers J, Hayes-Klug J, Langenberg J, Dahmes SJ et al (2009) Infectious prions in pre-clinical deer and transmission of chronic wasting disease solely by environmental exposure. PLoS One 4(6):e5916

Mathiason CK, Powers JG, Dahmes SJ, Osborn DA, Miller KV, Warren $\mathrm{RJ}$ et al (2006) Infectious prions in the saliva and blood of deer with chronic wasting disease. Science 314(5796):133-136

McNulty E, Nalls AV, Mellentine S, Hughes E, Pulscher L, Hoover EA et al (2019) Comparison of conventional, amplification and bioassay detection methods for a chronic wasting disease inoculum pool. PLoS One 14(5):e0216621
McNulty EE, Nalls AV, Xun R, Denkers ND, Hoover EA, Mathiason CK (2020) In vitro detection of haematogenous prions in whitetailed deer orally dosed with low concentrations of chronic wasting disease. J Gen Virol 101(3):347-361

Miller MW, Wild MA, Williams ES (1998) Epidemiology of chronic wasting disease in captive Rocky Mountain elk. J Wildl Dis 34(3):532-538

Miller MW, Williams ES (2003) Prion disease: horizontal prion transmission in mule deer. Nature 425(6953):35-36

Miller MW, Williams ES, Hobbs NT, Wolfe LL (2004) Environmental sources of prion transmission in mule deer. Emerg Infect Dis 10(6):1003-1006

Miller MW, Williams ES, McCarty CW, Spraker TR, Kreeger TJ, Larsen CT et al (2000) Epizootiology of chronic wasting disease in free-ranging cervids in Colorado and Wyoming. J Wildl Dis 36(4):676-690

Mitchell GB, Sigurdson CJ, O'Rourke KI, Algire J, Harrington NP, Walther I et al (2012) Experimental oral transmission of chronic wasting disease to reindeer (Rangifer tarandus tarandus). PLoS One 7(6):e39055

Monello RJ, Galloway NL, Powers JG, Madsen-Bouterse SA, Edwards WH, Wood ME et al (2017) Pathogen-mediated selection in freeranging elk populations infected by chronic wasting disease. Proc Natl Acad Sci U S A 114(46):12208-12212

Moore SJ, Vrentas CE, Hwang S, West Greenlee MH, Nicholson EM, Greenlee JJ (2018) Pathologic and biochemical characterization of $\operatorname{PrP}(\mathrm{Sc})$ from elk with PRNP polymorphisms at codon $132 \mathrm{after}$ experimental infection with the chronic wasting disease agent. BMC Vet Res 14(1):80

Nalls A, McNulty E, Powers J, Seelig D, Hoover C, Haley N et al (2013) Mother to offspring transmission of chronic wasting disease in Reeve's Muntjac deer. Prion 7:13

Nalls AV, McNulty E, Hoover CE, Pulscher LA, Hoover EA, Mathiason CK (2017) Infectious prions in the pregnancy microenvironment of CWD-infected Reeves' muntjac deer. J Virol 91(15):e00501-e517

Nalls AV, McNulty EE, Mayfield A, Crum JM, Keel MK, Hoover EA et al (2021) Detection of Chronic Wasting Disease prions in fetal tissues of free-ranging white-tailed deer. Viruses 13(12):2430

Nichols TA, Spraker TR, Rigg TD, Meyerett-Reid C, Hoover C, Michel $\mathrm{B}$ et al (2013) Intranasal inoculation of white-tailed deer (Odocoileus virginianus) with lyophilized chronic wasting disease prion particulate complexed to montmorillonite clay. PLoS One 8(5):e62455

O'Rourke KI, Baszler TV, Miller JM, Spraker TR, Sadler-Riggleman I, Knowles DP (1998) Monoclonal antibody F89/160.1.5 defines a conserved epitope on the ruminant prion protein. J Clin Microbiol 36(6): 1750-1755

Otero A, Velasquez CD, Aiken J, McKenzie D (2021) Chronic wasting disease: a cervid prion infection looming to spillover. Vet Res 52(1): 115

Peretz D, Williamson RA, Kaneko K, Vergara J, Leclerc E, SchmittUlms G et al (2001) Antibodies inhibit prion propagation and clear cell cultures of prion infectivity. Nature 412(6848):739-743

Perry VH (2004) The influence of systemic inflammation on inflammation in the brain: implications for chronic neurodegenerative disease. Brain Behav Immun 18(5):407-413

Plummer IH, Wright SD, Johnson CJ, Pedersen JA, Samuel MD (2017) Temporal patterns of chronic wasting disease prion excretion in three cervid species. J Gen Virol

Polymenidou M, Heppner FL, Pellicioli EC, Urich E, Miele G, Braun N et al (2004) Humoral immune response to native eukaryotic prion protein correlates with anti-prion protection. Proc Natl Acad Sci U S A 101(Suppl 2):14670-14676

Prusiner SB (1982) Novel proteinaceous infectious particles cause scrapie. Science 216(4542):136-144 
Ricci A AA, Bolton D, Chemaly M, Davies R, Salvador P, Escamez F, Girones R, Herman L, Koutsoumanis K, Lindqvist R, Norrung B, Robertson L, Sanaa M, Skandamis R, Snary E, Speybroeck N, Ter B, Kuile R, Threlfall J, Wahlstrom H, Benestad S, Gavier-Widen D, Miller M, Ru G, Telling G, Tryland M, Ortiz Pelaez A, Simmons M (2017) Chronic wasting disease (CWD) in cervids. EFSA J 15(1)

Rivera NA, Brandt AL, Novakofski JE, Mateus-Pinilla NE (2019) Chronic wasting disease in cervids: Prevalence, impact and management strategies. Vet Med (Auckl) 10:123-139

Robinson SJ, Samuel MD, O'Rourke KI, Johnson CJ (2012) The role of genetics in chronic wasting disease of North American cervids. Prion 6(2):153-162

Saa P, Castilla J, Soto C (2005) Cyclic amplification of protein misfolding and aggregation. Methods Mol Biol 299:53-65

Selariu A, Powers JG, Nalls A, Brandhuber M, Mayfield A, Fullaway S et al (2015) In utero transmission and tissue distribution of chronic wasting disease-associated prions in free-ranging Rocky Mountain elk. J Gen Virol

Service USFaW (2016) In: Edited by National Survey of Fishing HaWAR. https://www.fws.gov/wsfrprograms/subpages/nationalsurvey/ nat_survey2016.pdf. Accessed 10 Nov 2021

Sigurdson CJ, Williams ES, Miller MW, Spraker TR, O'Rourke KI, Hoover EA (1999) Oral transmission and early lymphoid tropism of chronic wasting disease PrPres in mule deer fawns (Odocoileus hemionus). J Gen Virol 80(Pt 10):2757-2764

Sohn HJ, Mitchell G, Lee YH, Kim HJ, Park KJ, Staskevicus A et al (2020) Experimental oral transmission of chronic wasting disease to sika deer (Cervus nippon). Prion 14(1):271-277

Soto P, Claflin IA, Bursott AL, Schwab-McCoy AD, Bartz JC (2021) Cellular prion protein gene polymorphisms linked to differential scrapie susceptibility correlate with distinct residue connectivity between secondary structure elements. J Biomol Struct Dyn 39(1):129-139

Spraker TR, Miller MW, Williams ES, Getzy DM, Adrian WJ, Schoonveld GG et al (1997) Spongiform encephalopathy in free-ranging mule deer (Odocoileus hemionus), white-tailed deer (Odocoileus virginianus) and Rocky Mountain elk (Cervus elaphus nelsoni) in northcentral Colorado. J Wildl Dis 33(1):1-6

Tamguney G, Miller MW, Giles K, Lemus A, Glidden DV, DeArmond SJ et al (2009) Transmission of scrapie and sheep-passaged bovine spongiform encephalopathy prions to transgenic mice expressing elk prion protein. J Gen Virol 90(Pt 4):1035-1047
Tamguney G, Richt JA, Hamir AN, Greenlee JJ, Miller MW, Wolfe LL et al (2012) Salivary prions in sheep and deer. Prion 6(1):52-61

Taschuk R, Scruten E, Woodbury M, Cashman N, Potter A, Griebel P et al (2017) Induction of $\operatorname{PrP}(\mathrm{Sc})$-specific systemic and mucosal immune responses in white-tailed deer with an oral vaccine for chronic wasting disease. Prion 11(5):368-380

Tennant JM, Li M, Henderson DM, Tyer ML, Denkers ND, Haley NJ et al (2020) Shedding and stability of CWD prion seeding activity in cervid feces. PLoS One 15(3):e0227094

Wang Z, Qin K, Camacho MV, Cali I, Yuan J, Shen P et al (2021) Generation of human chronic wasting disease in transgenic mice. Acta Neuropathol Commun 9(1):158

Wells GA, Hawkins SA, Green RB, Austin AR, Dexter I, Spencer YI et al (1998) Preliminary observations on the pathogenesis of experimental bovine spongiform encephalopathy (BSE): an update. Vet Rec 142(5):103-106

White AR, Enever P, Tayebi M, Mushens R, Linehan J, Brandner S et al (2003) Monoclonal antibodies inhibit prion replication and delay the development of prion disease. Nature 422(6927):80-83

Wild MA, Spraker TR, Sigurdson CJ, O'Rourke KI, Miller MW (2002) Preclinical diagnosis of chronic wasting disease in captive mule deer (Odocoileus hemionus) and white-tailed deer (Odocoileus virginianus) using tonsillar biopsy. J Gen Virol 83(Pt 10):2629-2634

Wille H, Requena JR (2018) The structure of PrP(Sc) prions. Pathogens 7(1)

Williams ES, Young S (1980) Chronic wasting disease of captive mule deer: a spongiform encephalopathy. J Wildl Dis 16(1):89-98

Williams ES, Young S (1992) Spongiform encephalopathies in Cervidae. Rev Sci Tech 11(2):551-567

Wood ME, Griebel P, Huizenga ML, Lockwood S, Hansen C, Potter A et al (2018) Accelerated onset of chronic wasting disease in elk (Cervus canadensis) vaccinated with a $\operatorname{PrP}(\mathrm{Sc})$-specific vaccine and housed in a prion contaminated environment. Vaccine 36(50):7737-7743

Publisher's Note Springer Nature remains neutral with regard to jurisdictional claims in published maps and institutional affiliations. 